\title{
At 11 months, prosody still outranks statistics
}

\section{Elizabeth K. Johnson ${ }^{1}$ and Amanda H. Seidl ${ }^{2}$}

1. Department of Psychology, University of Toronto, Canada

2. Purdue University, W. Lafayette, Indiana, USA

\begin{abstract}
English-learning 7.5-month-olds are heavily biased to perceive stressed syllables as word onsets. By 11 months, however, infants begin segmenting non-initially stressed words from speech. Using the same artificial language methodology as Johnson and Jusczyk (2001), we explored the possibility that the emergence of this ability is linked to a decreased reliance on prosodic cues to word boundaries accompanied by an increased reliance on syllable distribution cues. In a baseline study, where only statistical cues to word boundaries were present, infants exhibited a familiarity preference for statistical words. When conflicting stress cues were added to the speech stream, infants exhibited a familiarity preference for stress as opposed to statistical words. This was interpreted as evidence that 11-month-olds weight stress cues to word boundaries more heavily than statistical cues. Experiment 2 further investigated these results with a language containing convergent cues to word boundaries. The results of Experiment 2 were not conclusive. A third experiment using new stimuli and a different experimental design supported the conclusion that 11-month-olds rely more heavily on prosodic than statistical cues to word boundaries. We conclude that the emergence of the ability to segment non-initially stressed words from speech is not likely to be tied to an increased reliance on syllable distribution cues relative to stress cues, but instead may emerge due to an increased reliance on and integration of a broad array of segmentation cues.
\end{abstract}

\section{Introduction}

Continuous speech contains no reliable cues to word boundaries (Cole \& Jakimik, 1980). Thus, finding words in speech is a significant challenge for infants. Despite the difficulties involved in learning to perceive speech as a string of discrete words, infants begin extracting word-forms from speech between 6 and 7.5 months of age (Bortfeld, Morgan, Golinkoff \& Rathbun, 2005; Jusczyk \& Aslin, 1995).

At 7.5 months of age, English-learning infants have been shown to use two strategies to find word boundaries in fluent speech. One segmentation strategy that infants use is prosodic in nature. Adult English speakers tend to perceive stressed syllables as word onsets (Cutler \& Butterfield, 1992). This segmentation strategy is effective, since $90 \%$ of content words in English begin with a stressed syllable (Cutler \& Carter, 1987). English-learning 7.5-montholds behave similarly in that they readily extract stressinitial bisyllables (e.g. doctor) but not stress-final bisyllables (e.g. guitar) from speech (Jusczyk, Houston \& Newsome, 1999b; see also Curtin, Mintz \& Christiansen, 2005). Another segmentation strategy that infants have been found to use is statistical ${ }^{1}$ in nature. Transitional probabilities between syllables, typically thought of as the statistical likelihood that two syllables will co-occur (Hayes \& Clark, 1970), provide probabilistic information to the location of word boundaries by highlighting peaks and troughs in the probability of syllable transitions in speech. If the frequency-weighted likelihood that one syllable will follow another in running speech is high, then the chances are high that the two syllables belong to the same word. Conversely, if the likelihood of cooccurrence is low, then chances are high that the syllables in question do not belong to the same word.

Artificial language learning experiments have provided evidence that 8-month-olds are able to segment an artificial language containing no cues to word boundaries other than the transitional probabilities between syllables (Saffran, Aslin \& Newport, 1996). Based on these and subsequent data from related studies, it has been argued that tracking transitional probabilities between syllables may be a language-general segmentation strategy that

\footnotetext{
${ }^{1}$ The term 'statistical' is used to describe a very specific segmentation strategy that involves tracking transitional probabilities between syllables. Use of this term is not meant to suggest that other segmentation strategies do not involve a statistical component.
}

Address for Correspondence: Elizabeth Johnson, Department of Psychology, University of Toronto Mississauga, Mississauga, ON, Canada L5L 1C6; e-mail: elizabeth.johnson@utoronto.ca

The authors of this paper are listed in alphabetical order. 
helps language learners extract their first words from speech (Thiessen \& Saffran, 2003).

Given that infants are sensitive to both prosodic and statistical cues to word boundaries at 7.5-8 months of age, it has been difficult to say for sure what strategy infants use to first begin segmenting words from speech. One hypothesis is that infants begin segmenting words from speech by tracking transitional probabilities between syllables. Once infants have segmented a large number of words using statistical cues, they are in a position to notice that the majority of English words have word-initial stress and can subsequently use this regularity of stress pattern to segment words (Thiessen \& Saffran, 2003, 2007; see Swingley, 2005, for discussion). Another hypothesis is that early on infants learn that stress signals word onsets by attending to isolated words and short utterances (Brent \& Siskind, 2001), and that transitional probabilities between syllables play a secondary role in infants' early segmentation success (Johnson \& Jusczyk, 2001; Jusczyk, 1997). Evidence for both hypotheses exists. Thiessen and Saffran (2003) demonstrated that 6-month-olds are able to segment an artificial language containing no cues to word boundaries other than transitional probabilities between syllables. Since infants at this age have not yet learned the contingency between word onsets and stress in English, Thiessen and Saffran argue that prosodic cues to word boundaries are most likely bootstrapped from the use of statistical cues. However, this line of evidence is weakened by the fact that 6-month-olds' ability to track transitional probabilities in an artificial language may not scale up to the challenge of real language. For example, the 6-month-olds in Thiessen and Saffran's study might have failed to segment the artificial language had it not consisted entirely of four bisyllabic CVCV words produced with very little phonetic variation (Tyler \& Johnson, 2006). Evidence for the contrasting view, i.e. the view that transitional probabilities may play a secondary role in early segmentation, comes from a study by Johnson and Jusczyk showing that 8-month-olds weight stress cues to word boundaries more heavily than statistical cues. These results lead Johnson and Jusczyk to argue that prosodic cues to word boundaries are probably not learned by tracking transitional probabilities between syllables. However, despite 8-month-olds' behavior, infants could easily begin segmenting words from speech using transitional probabilities between syllables and then re-rank their reliance on stress versus statistical cues shortly after learning that stress tends to signal word onsets.

The debate over how infants first begin their segmentation of words from speech continues. In this paper we do not add further to the debate on initial cue reliance. Rather, we focus on later development, investigating how 11-month-olds overcome their reliance on metrical structure to arrive at a segmentation strategy that will function for the segmentation of both initially stressed (trochaic) and non-initially stressed (iambic) words. Because English contains a sizeable minority of iambic words (e.g. many verbs) as well as stressed monosyllables followed by unstressed function words (e.g. ...cat is), this is an important piece of the puzzle for understanding the development of word segmentation.

As discussed above, at 7.5-8 months of age, Englishlearning infants perceive syllables carrying primary stress as word onsets. This holds for both artificial language studies (Curtin et al., 2005; Johnson \& Jusczyk, 2001; Thiessen \& Saffran, 2003) as well as natural language studies (Jusczyk et al., 1999b). However, by 11 months, English-learning infants are proficient at segmenting both iambic and trochaic nouns from speech (Jusczyk et al., 1999b; Johnson, 2005). Thus, some time between 7.5 and 11 months of age (an age when infants' speech processing skills are rapidly developing; Kuhl, 2000; Werker \& Tees, 1984), infants abandon their over-reliance on stress cues to word boundaries in favor of a strategy that allows them to segment both trochaic and iambic words from speech. What changes in infants' speech processing abilities could underlie this crucial development? One possibility is that infants' growing awareness of multiple cues to word boundaries and perhaps their ability to treat cues separately (Seidl \& Cristià, 2008) may play a critical role in the emergence of their ability to segment iambic words from speech. However, the precise mechanism underlying the evolution of infants' ability to segment non-initially stressed words from speech is not well understood. In particular, we do not know whether older infants are integrating multiple cues (allophonic, phonotactic, stress, statistical) with growing lexical knowledge, or whether their increased ability to segment non-initially stressed words is just due to an increased reliance on statistical cues accompanied by a decreased reliance on stress cues. In this paper, we investigate the relative ranking of statistical and stress cues at this older age.

This hypothetical re-ranking of stress and statistical cues may be related to an increase in infants' reliance on statistical cues. This increased reliance on statistical cues could simply be driven by the extra 3.5 months of language exposure experienced by 11- as opposed to 8-month-olds. It also seems possible that either the integration or separation of multiple segmentation cues might lead infants to realize that stress is not an infallible indicator of word onsets. In terms of the integration of cues, by 11 months infants have learned about both phonotactic (Jusczyk, Luce \& Charles-Luce, 1994; Mattys \& Jusczyk, 2001) and allophonic cues (Jusczyk, Hohne \& Bauman, 1999a) to word boundaries. If infants notice that stress cues sometimes can conflict with these other cues to word boundaries, then this could drive them to rely more heavily on transitional probabilities between word boundaries rather than lexical stress. This prosody/statistics cue re-ranking hypothesis for explaining how infants first begin segmenting iambic words from speech is well articulated by Thiessen and Saffran (2003, p. 17): 
By 8 months, infants are attending primarily to stress as a cue to word segmentation, treating stressed syllables as word onsets even when that information conflicts with statistical information . . by 10.5 months, infants may have discovered that stress cues correlate less well with multiple other cues to word segmentation than do statistical cues, and they thus favor statistical cues when the two are placed in conflict.

Furthermore, the latter possibility (that the re-ranking is mainly driven by a reduction in the attention to stress cues) fits well with reports that adults (McQueen, 1998), in contrast to 9-month-olds (Mattys, Jusczyk, Luce \& Morgan, 1999), weight phonotactic cues to word boundaries more heavily than stress cues. In this paper, we use the artificial language methodology to explore the possibility that 11-month-olds weight syllable distribution cues to word boundaries more heavily than stress cues. If so, such a change in the relative weighting of statistical versus prosodic cues to word boundaries at 11 months would represent an important developmental milestone, and provide an important clue to understanding the mechanisms underlying the maturation of infants' early word segmentation strategies.

\section{Experiment 1}

In Experiment 1a, infants were familiarized with an artificial language containing four trisyllabic words, and no cues to word boundaries other than the statistical structure of the language. Following familiarization, infants were tested for their orientation preference for statistical words versus statistical partwords. If 11-month-olds are able to extract words from the artificial language by tracking the transitional probabilities between syllables, then we predicted that they would orient differentially to statistical partwords versus statistical whole words. A novelty preference for statistical partwords, as observed with 8-montholds by Johnson and Jusczyk (2001), was the most likely outcome. However, given the difficulties inherent in predicting the direction of preferences in artificial language studies (Gerken \& Zamuner, 2004; Thiessen, Hill \& Saffran, 2005; Thiessen \& Saffran, 2003), a familiarity preference for statistical words would not be entirely unexpected, and may even indicate something about the level of difficulty of this kind of task for this age group since we predict familiarity effects for more difficult tasks and novelty effects for simpler tasks (Hunter \& Ames, 1988). Regardless of the direction of preference, in this paradigm, the crucial point is that any orientation preference for one test item type over another is evidence for segmentation of the language.

In Experiment 1b, word-final stress was added to the statistical words in the speech stream. Since Englishlearning infants tend to perceive stressed syllables as word onsets, the language used in Experiment $1 \mathrm{~b}$ contains conflicting stress and statistical cues. If 11-month-olds, like 8-month-olds, weight stress cues to word boundaries more heavily than statistical cues, then they should reverse their orientation preferences, since they would now segment the speech stream according to stress cues. If, on the other hand, 11-month-olds differ from 8-month-olds in that they weight statistical cues to word boundaries more heavily than stress cues, then they should segment the speech stream in the exact same way as they did in Experiment 1a. Note that the key prediction here is that the addition of stress would either have no effect on how infants segment the language (suggesting that older infants weight transitional probabilities between syllables more heavily than stress cues), or the addition of stress would cause infants to reverse the way they segment the language (suggesting that infants weight stress cues more heavily than syllable-distribution cues).

\section{Methods}

\section{Participants}

Forty English-learning 11-month-olds from the greater Lafayette, IN area were tested (20 females; 20 males). The approximate age of the infants was 11 months, with a mean age of 335 days (range: 10.25 to 11.5 months). The data from 15 additional infants were excluded due to fussiness (10), sleepiness (3) or parental interference (2). Parental consent was obtained for all participants.

\section{Stimuli}

Experiments $1 \mathrm{a}$ and $1 \mathrm{~b}$ used the exact same stimuli as Johnson and Jusczyk (2001). The stimuli for Experiment 1a were constructed out of $12 \mathrm{CV}$ syllables that were recorded in isolation by a female speaker. Acoustic analyses were carried out to ensure that the syllables were closely matched in f0 $(M=268 \mathrm{~Hz} ; S D=3.6 \mathrm{~Hz})$, amplitude $(M=57 \mathrm{~dB} ; S D=3 \mathrm{~dB})$, and duration $(M=273 \mathrm{~ms}$; $S D=22 \mathrm{~ms})$. The syllables were concatenated into two sets of four trisyllabic words (Language A: golatu, daropi, pabiku, tibudo; Language B: tudaro, pigola, bikuti, budopa). ${ }^{2}$ Each of the two 2.5-minute speech streams used during the familiarization phase of Experiment 1a was created by concatenating the words of each language together in a pseudo-random order with the stipulation that no single word occurred twice in a row, and no pauses occurred between syllables or words. Since no syllable occurred in more than one word in either language, the transitional probabilities within words was always 1.0 whereas the transitional probabilities between words was always equal to .33. Statistical words occurred 45 times, and statistical partwords occurred 15 times.

\footnotetext{
${ }^{2}$ As noted by an anonymous reviewer, the syllables were similar but not identical in length and pitch. Thus some syllables may have sounded more like word onsets than other syllables. We controlled for this potential confound by using the same syllable recordings to create both Language A and Language B. Syllables that occurred in onset position of Language A never occurred in onset position of Language B.
} 
The languages used in Experiment $1 \mathrm{~b}$ were identical in all respects to the languages used in Experiment 1a except for the addition of conflicting stress cues to word boundaries. Each time a partword test item appeared in the familiarization sequence, its first syllable was replaced with a stressed version, which was higher in pitch $(M=$ $287 \mathrm{~Hz})$, and amplitude $(M=65 \mathrm{~dB})$, and longer in duration $(M=306 \mathrm{~ms})$ than its unstressed counterpart. This resulted in the addition of 30 stressed syllables to the speech stream. An orthographic representation of the resulting speech stream would appear as follows (boldface indicates stress): . . . daroPIgolatutibudopabikugolaTUdaropipa bikutibudo...., where daropi and golatu are statistical words, and PIgola and TUdaro are statistical partwords.

Four test items were constructed out of the same 12 unstressed syllables used to make the familiarization languages. Partword test items consisted of the last syllable of one word plus the first two syllables of another word (e.g. tudaro was formed from golatu and daropi). Note that the words of Language A formed partwords in Language $B$, and vice versa, so that all infants could be tested on the same four test items (pabiku, tibudo, tudaro, pigola).

\section{Design}

All infants were randomly assigned to familiarization with one of two streams of speech: Language A or Language B. The infants in Experiment 1a heard a speech stream containing no cues to word boundaries other than the statistical structure of the language, whereas the infants in Experiment 1b heard a speech stream containing conflicting stress and statistical cues to word boundaries.

\section{Procedure and apparatus}

Infants were tested using the same version of the Headturn Preference procedure used in Johnson and Jusczyk (2001) and Saffran et al. (1996). Infants sat on the lap of a caregiver in the center of a three-sided booth. The experimenter remained out of view of the infant, recording the direction and duration of the infants' orientation through the use of a button box. The randomization of stimuli and termination of trials was computer-controlled. A red light and a speaker were mounted at eye level on the center of each side panel, and a green light was located at eye level on the center of the front panel. During the familiarization phase, the green light flashed at the start of each trial. Once the infant oriented towards this light, the familiarization speech stream played from both speakers continuously until the end of the sound file was reached. Throughout the familiarization, the lights in the booth were lit and extinguished contingent upon the infant's headturns.

The test phase immediately followed the familiarization phase. Each of the 12 test trials (three trials for each of the four test items) began with the blinking center light.
Once the infant oriented toward the green light, it stopped blinking and one of the two side lights began blinking. Once the infant oriented toward the blinking light, a test item was repeated with a $500 \mathrm{~ms}$ ISI until the infant looked away for more than 2 consecutive seconds or until 15 repetitions of the test item had occurred. Test trials were blocked and presented in random order within those blocks. Half of the trials consisted of repetitions of statistical words; the other half consisted of repetitions of statistical partwords. All recordings were played at a comfortable listening level (approximately $68 \mathrm{~dB}$ ).

Both the caregiver and the experimenter listened to loud masking music mixed with low level white noise and wore tight-fitting Peltor aviation headphones. The dependent measure in this study was orientation times to test stimuli.

\section{Results and discussion}

We will first examine the results of Experiment 1a (statistical cues only). Mean orientation times to statistical words and statistical partwords were calculated for each of the 20 participants (see Figure 1, Experiment 1a). Eighteen out of the 20 infants listened longer to the statistical words than to the statistical partwords. This difference was significant by a sign test, $p=.004$. On average, infants oriented to the statistical words for 8.06 seconds $(S D=2.47)$ and the statistical partwords for 7.08 seconds $(S D=2.8)$. A 2 (Group: Language A vs. Language B) $\times 2$ (Test Item Type: statistical word vs. statistical partword) mixed design ANOVA revealed a main effect of Test Item Type, $F(1,18)=7.76, p<.05$, but no main effect of Group, $F(1,18)<1$, or interaction between Group and Test Item Type, $F(1,18)<1$.

The infants in Experiment 1a showed a familiarity preference for statistical words over statistical partwords, indicating their ability to segment an artificial language containing no cues to word boundaries other than transitional probabilities between syllables. Note that the 8-month-olds tested by Johnson and Jusczyk (2001) with

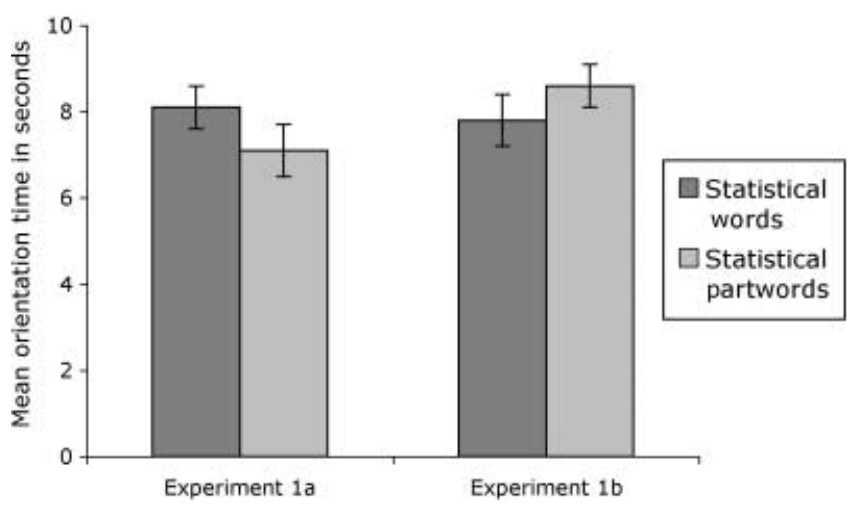

Figure 1 Mean orientation in seconds to statistical words and statistical partwords for Experiment 1. Error bars indicate Standard Error. 
the same stimuli showed a novelty preference. Since the materials were identical, the difference in age between subjects tested in these experiments is the most likely explanation for the 11-month-olds' familiarity as opposed to novelty preference. It is unusual for older infants to display a familiarity preference while younger infants display a novelty preference (Hunter \& Ames, 1988). But since it is likely that the 11-month-olds in the current study typically rely on a wider variety of cues to word boundaries than 8-month-olds, the task may have been more difficult for them than for 8-month-olds who typically rely on a subset of cues. Fitting with this explanation, Thiessen and Saffran (2003) reported that the older infants in their study also exhibited a familiarity preference for statistical words. Nonetheless, the important finding here is that infants responded differentially to statistical words and partwords, indicating that they had successfully segmented the language.

Next we will examine the results of Experiment $1 \mathrm{~b}$ (statistical vs. stress cues). Mean orientation times to statistical words and statistical partwords were calculated for each of the 20 participants (see Figure 1, Experiment 1b). Fifteen out of the 20 infants listened longer to the statistical partwords than to the statistical words. This difference was significant by a sign test, $p=.04$. Infants oriented to the statistical partwords for 8.60 seconds $(S D=2.5)$ and the statistical words for 7.79 seconds $(S D=2.7)$. A 2 (Group: Language A vs. Language B) $\times 2$ (Test Item Type: statistical word vs. statistical partword) mixed design ANOVA revealed a significant effect of Test Item Type, $F(1,18)=4.82, p<.05$, but no main effect of Group, $F(1,18)=1.8, p>.1$, or interaction between Group and Test Item Type, $F(1,18)<1$.

Finally, a 2 (Experimental: Experiment 1a vs. Experiment $1 b) \times 2$ (Test Item Type: statistical word vs. statistical partword) mixed design ANOVA was carried out to compare the results of Experiments 1a and 1b. This analysis revealed a significant interaction between Experiment and Test Item Type, $F(1,38)=12.97, p<.01$, but no main effects of Test Item Type or Experiment, $F s(1,42)<1$.

Infants in Experiment $1 \mathrm{~b}$ oriented longer to statistical partwords (or stress words) than to statistical words, exhibiting the opposite listening preference observed in Experiment 1a. Assuming a familiarity preference in both Experiment 1a and 1b, this finding demonstrates that the infants tested in Experiment 1a extracted words defined by statistical cues whereas the infants tested in Experiment $1 \mathrm{~b}$ extracted words defined by stress cues. In short, 11-month-olds weight stress cues to word boundaries more heavily than statistical cues to word boundaries.

\section{Experiment 2}

Our interpretation of Experiment 1 is dependent on the assumption that the infants in both Experiment 1a and $1 \mathrm{~b}$ are displaying a preference for what they perceive as familiar. But perhaps the added complexity of the familiarization languages used in Experiment $1 \mathrm{~b}$ simply made the segmentation task more difficult, causing infants to display a novelty as opposed to a familiarity preference. Major changes to the complexity of an artificial language have indeed caused directional shifts in infants' orientation preferences (Thiessen \& Saffran, 2003). However, this seems an unlikely explanation for our results for two reasons. First, the direction of the preference shift: Novelty preferences are associated with easier, not more complex tasks (Hunter \& Ames, 1988). It seems unlikely that the presence of conflicting cues would cause infants to shift to a novelty preference. Second, past studies have shown that adding a naturalistic speech cue (e.g. coarticulation) to a small subset of words in an artificial language does not on its own result in a shift in the direction of orientation preferences (see control Experiment 4 in Johnson \& Jusczyk, 2001). Nonetheless, Experiment 2 examines this issue by shifting the location of the stressed syllables to word-initial as opposed to word-final position. In this way, infants are familiarized with a language containing no conflicting stress cues to word boundaries (as in Experiment 1a), but the familiarization language is matched for complexity with the language used in Experiment $1 \mathrm{~b}$. If our interpretation of Experiment 1 is correct, then the results of the current experiment should resemble those of Experiment 1a, i.e. longer looking times to statistical words. If, on the other hand, the switch in preferences observed in Experiment 1 was due to the added complexity of the familiarization language, then the results of Experiment 2 should resemble Experiment 1b, i.e. longer looking times towards statistical partwords.

\section{Methods}

\section{Participants}

Twenty-four English-learning 11-month-olds from the greater Lafayette, IN area were tested (10 females; 12 males). The approximate age of the infants was 11 months, with a mean age of 324 days (range: 10.25 to 11.5 months). The data from 6 additional infants were excluded due to fussiness. Parental consent was obtained for all participants.

\section{Stimuli}

Experiment 2 used the same syllable tokens as Experiment 1. As in Experiment 1b, the language used in Experiment 2 contained both stress and statistical cues to word boundaries. In both experiments 15 tokens of each of two stressed syllables were added to the speech stream. The primary difference between Experiment $1 \mathrm{~b}$ and Experiment 2, however, was the placement of the stressed syllables. In Experiment $1 \mathrm{~b}$ the stress cues conflicted with the statistical cues, i.e. the stressed syllables were situated in word-final position. In Experiment 2, the stress cues coincided with statistical cues, i.e. they occurred in word-initial position. An orthographic representation 
of the speech stream would appear as follows (boldface indicates stress placement):... GOlatutibudopabikuGO latuDAropi . . . , where DAropi and GOlatu are statistical words, and pigola and tudaro are statistical partwords.

\section{Design}

Aside from the location of stress cues, all other aspects of the experimental design were identical to Experiment $1 b$.

\section{Procedure and apparatus}

The same procedure and apparatus were used as in Experiment 1.

\section{Results and discussion}

Mean orientation times to statistical words and statistical partwords were calculated for each of the 24 participants (see Figure 2). Eleven out of the 24 infants listened longer to the statistical words than to the statistical partwords. This difference was not significant by a sign test, $p=.84$. On average, infants oriented to the statistical words for 8.07 seconds $(S D=2.9)$ and to the statistical partwords for 8.04 seconds $(S D=2.6)$. A 2 (Group: Language A vs. Language B) $\times 2$ (Test Item Type: statistical word vs. statistical partword) mixed design ANOVA revealed that neither of the main effects nor any of the interactions were significant, $F s(1,22)<1$.

These results are somewhat unexpected. We had predicted that if the preference switch in Experiment 1a and $1 \mathrm{~b}$ were simply due to the added complexity of the familiarization stream, then the results of Experiment 2 should resemble those of Experiment 1b, i.e. infants should listen longer to statistical partwords than words. If, however, the preference switch was due to infants' use of stress to identify word boundaries, then the results of Experiment 2 should resemble those of Experiment 1a, i.e. infants should listen longer to statistical words than statistical partwords. Our results did not fit with either of these predictions. Instead, infants failed to demonstrate

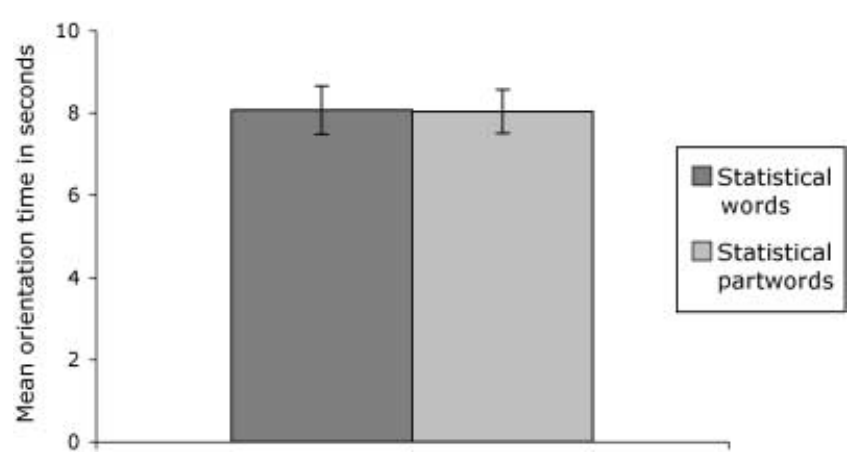

Figure 2 Mean orientation in seconds to statistical words and statistical partwords for Experiment 2. Error bars indicate Standard Error. any looking preferences. On the one hand, this demonstrates that the presence of stressed syllables in the familiarization speech stream does not make infants reverse their orientation preferences. This suggests that the change in preference found in Experiment $1 \mathrm{~b}$ was not simply due to the added complexity of the familiarization language. On the other hand, infants displayed no looking time preference at all. Why would a change in the location of stress to word-initial as opposed to word-final position obliterate infants' orientation preferences? What we know about infants' tendency to display novelty versus familiarity preferences provides a possible answer to this question: When infants are over-trained and a task gets simpler, infants tend to become bored and exhibit a novelty preference during testing. The artificial language used in Experiment $1 \mathrm{~b}$ contained conflicting cues to word boundaries, but the artificial language used in Experiment 2 contained coincident cues to word boundaries. Since the segmentation task was easier in Experiment 2 than in Experiment 1b, infants' tendency to exhibit a familiarity preference may have begun to shift towards a tendency to exhibit a novelty preference. The lack of orientation preference may have resulted because the task was too easy to elicit a familiarity preference, but not quite easy enough to elicit a novelty preference. If this interpretation of Experiment 2 is accurate, then infants' failure to exhibit any orientation preference in Experiment 2 not only provides support for our interpretation of Experiment 1, it also further confirms that infants use both statistical and stress cues to find word boundaries in speech.

But how can we confirm whether or not our interpretation of Experiment 2 is accurate? Null results are notoriously difficult to interpret in infant studies (Aslin, 2007). One approach to this dilemma would be to both shorten and lengthen the duration of the language familiarization phase and observe whether the shorter exposure leads to a familiarity preference whereas the longer exposure leads to a novelty preference (see Thiessen et al., 2005). Or we could add stress to the onset of every word rather than every third instance of two out of the four words. But, for several reasons, neither of these manipulations is optimal. First, it is difficult to predict how much or how little exposure would be needed to elicit a novelty/familiarity preference. Second, increasing exposure duration leads to increased dropout rates, which in turn leads to a more selective subject sample. Third, in order to allow comparison with Experiment 1, we would have to rerun subjects in that experiment with comparable length of exposure or strength of prosodic cues.

For all of the reasons mentioned in the previous paragraph, we sought out a more effective means for testing whether 11-month-olds weight prosodic cues more heavily than statistical cues. Using an artificial language design similar to that used by Thiessen and Saffran (2003) seemed like the best option for addressing our question of interest. The artificial language design used by Thiessen and Saffran (2003) differed from that used by Johnson 
and Jusczyk in that it controlled for two potential confounds: (1) differences in the similarity between familiarization words and test items across conditions, and (2) differences in the complexity of the speech streams used in speech-plus-statistics versus the baseline familiarization. Thus, in Experiment 3 we have chosen to use Thiessen and Saffran's methodology to further test 11-month-olds' reliance on stress versus statistical cues to word boundaries.

\section{Experiment 3}

In Experiment 1a, we familiarized 11-month-olds with an artificial language containing no cues to word boundaries other than the transitional probabilities between syllables. Infants listening longer to statistical words during a subsequent test phase indicated their ability to use statistical cues to find word boundaries. In Experiment 1b, we familiarized 11-month-olds to the same artificial language, but we added conflicting stress cues to the speech stream. In this case, infants listened longer to statistical partwords during a subsequent test phase. We interpreted this as evidence that 11-month-olds behave in a way similar to 8- and 9-month-olds in that they weight stress cues to word boundaries more heavily than statistical cues. However, there are alternative explanations for our results if one questions whether or not infants in Experiments 1a and 1b were all demonstrating a familiarity preference for words they recognized as opposed to a familiarity preference for words in Experiment 1a and a novelty preference for partwords in Experiment $1 \mathrm{~b}$. The speech stream used in Experiment 1b, i.e. the speech stream containing conflicting stress and statistical cues to word boundaries, was in fact more complicated than the speech stream used in Experiment 1a. Moreover, since the familiarization stream in Experiment 1a contained no stressed syllables, there was a greater acoustic difference between the familiarization syllables and test syllables in Experiment $1 \mathrm{~b}$ than in Experiment 1a. It could be argued that either one of these factors could have caused infants to switch their looking time preferences. We have given two main reasons why these alternative explanations are unsatisfactory. First, the direction of change does not make sense. Why would infants switch to a novelty preference when the language was made more difficult to learn? Second, a control experiment run by Johnson and Jusczyk (2001) suggests that these types of small changes do not cause infants to switch their looking time preferences. We ran Experiment 2 in an attempt to gain further support for our interpretation of Experiment 1. However, due to difficulties in interpreting direction of preferences, the results of Experiment 2 were inconclusive.

In the current experiment, we tried to replicate the findings we reported in Experiment 1 with a new set of stimuli that controlled for the two confounds mentioned above. Infants tested in Experiment 3 heard a language containing four bisyllabic words. For half of the infants, the familiarization words contained words stressed on the first syllable. For the other half the familiarization stream contained words stressed on the second syllable. All infants were tested on the same four test items: two statistical words and two statistical partwords. The test items were neither iambic nor trochaic because the component syllables were produced in isolation and spliced together. This design controls for the confounds present in Experiment 1 since both familiarizations are equally complex and all infants were tested on words that are equally distinct from the familiarization stream. If our interpretation of Experiment 1 is correct, we make the following predictions. Infants in the trochaic familiarization condition should look longer to familiar statistical words (which also happen to be words with trochaic stress, since stress and statistical cues are convergent in this language). Infants in the iambic familiarization condition, however, should look longer to familiar prosodic or trochaic words (which happen to be statistical partwords). In short, if 11-month-olds truly rely more heavily on stress cues than syllable distribution cues, then they should always prefer to listen to the words that carry word-initial stress in the familiarization stream. On the other hand, if the results of Experiment 1 were simply due to differences between the two familiarization streams (stress present versus no stress present) and 11-month-olds actually weight syllable distribution cues to word boundaries more heavily than stress cues, then we would expect that infants in the current experiment would always extract the same words regardless of stress placement in the training phase.

\section{Methods}

\section{Participants}

Thirty English-learning 11-month-olds from the greater Lafayette, IN area were tested (17 females; 13 males). Approximate age was 11 months old, with a mean age of 330 days (range: 10.5 to 11.5 months). The data from 4 additional infants were excluded due to fussiness or parental interference. Parental consent was obtained for all participants.

\section{Stimuli}

New stimuli were created for use in Experiment 3. The artificial language used in this study had the same structure as the language used by Thiessen and Saffran (2003, 2004). As in Thiessen and Saffran's stimuli, we created two languages containing four bisyllabic CVCV words (dimu, ludo, baro, and gafi). The segmental structure of words was identical in both languages, and the only difference was the placement of stress with respect to word boundaries. All words in the trochaic language carried word-initial stress whereas all words in the iambic language carried word-final stress. The speech stream contained 60 tokens of baro, 60 tokens of dimu, 30 tokens of ludo, and 30 
tokens of gafi. As in Thiessen and Saffran, two of the words occurred twice as often as the other two. Using this design allowed us to test infants on words and partwords that occurred equally frequently in the familiarization (test words for all infants were ludo and gafi, test partwords were $m u b a$ and rodi). This ensured that infants' looking time differences were driven by their use of transitional probabilities rather than simple co-occurrence frequencies (see Aslin, Saffran \& Newport, 1998, for discussion). Since no syllable occurred in more than one word in either language, the transitional probabilities within words was always 1.0 whereas the transitional probabilities between words was less than .4 .

In contrast to Thiessen and Saffran (2003), we chose to build our language out of natural syllable recordings rather than those produced by a speech synthesizer. We also chose to generate our stimuli with a different procedure than that used in Johnson and Jusczyk (2001). These decisions were in large part driven by our desire to create clearly enunciated stimuli with natural stress and coarticulation. Note that Thiessen and Saffran, as well as Johnson and Jusczyk, created stressed syllables in an artificial manner whereas the artificial language used in the current study had naturally stressed syllables. Thus, the stimuli used in the current study were arguably more ecologically valid than the stimuli used in past infant artificial language learning studies. Since it is impossible for a human to speak for long stretches of time without pausing for breath, we had to construct our 1.5-minute familiarization speech-stream by splicing together parts of shorter utterances. We chose not to create our language out of syllables recorded in isolation (as in Johnson \& Juscyzk, 2001) because doing so results in an unnatural lack of coarticulation between syllables. Thus, we recorded our stimuli using a procedure very similar to that used by Curtin et al. (2005). A female native English speaker recorded each token of the words in the language embedded within a five-syllable nonsense word (e.g. the word gafi was produced as part of the longer nonsense word barogafidi). All nonsense carrier words were spoken with the prosodic shape of the five-syllable word unbelievable (boldface indicates primary lexical stress). The target CVCV words were spliced out from the longer nonsense words at the zero crossings, and concatenated together to create a continuous speech stream. For example, in order to create the three-word stretch of speech barogafidimu for the trochaic language, three five-syllable nonsense words were recorded: dimubaroga, barogafidi, and gafidimulu. Baro was spliced from the first word, gafi from the second, and dimu from the third. Note that because of the context these words were spliced from, natural sounding coarticulation resulted when the target words were strung together. Note that since all syllables were equally coarticulated, degree of coarticulation could not serve as a word segmentation cue. In order to create the same three-word stretch of speech for the iambic language, three different five-syllable nonsense words were had to be recorded: mubarogafi, rogafidimu, and fidimuludo. Acoustic analyses confirmed that on average word-initial syllables were longer in duration, higher in pitch, and greater in amplitude in the trochaic language than the iambic language: trochaic language: $260 \mathrm{~ms}$, $299 \mathrm{~Hz}, 74 \mathrm{~dB}$; iambic language: $206 \mathrm{~ms}, 191 \mathrm{~Hz}, 69 \mathrm{~dB}$. Likewise, the word-final syllables were on average longer in duration, higher in pitch, and greater in amplitude in the iambic language than in the trochaic language: Trochaic language: $215 \mathrm{~ms}, 162 \mathrm{~Hz}, 68 \mathrm{~dB}$; iambic language: $279 \mathrm{~ms}, 352 \mathrm{~Hz}, 75 \mathrm{~dB}$. The four test items were created by recording individual syllables in isolation and stringing them together. The syllables were recorded in isolation to ensure that they would have equal prominence, i.e. the test items were created out of two stressed syllables and were thus neither iambic or trochaic (syllable 1 mean vowel length $=184 \mathrm{~ms}$; syllable 2 mean vowel length $=$ $193 \mathrm{~ms}$; syllable 1 mean pitch $=167 \mathrm{~Hz}$; syllable 2 mean pitch $=169 \mathrm{~Hz}$; syllable 1 mean amplitude $=72 \mathrm{~dB}$; syllable 2 mean amplitude $=71 \mathrm{~dB}$ ).

\section{Design}

Half of the infants were randomly assigned to hear the trochaic language, and the other half were assigned to hear the iambic language. The words in both languages were identical; the only difference between the trochaic and iambic language was whether the words carried word-initial or word-final stress. All infants were tested on the same four test items: two words (gafi and ludo) and two partwords (rodi and muba).

\section{Procedure and apparatus}

The same procedure and apparatus were used as in Experiment 1.

\section{Results and discussion}

Mean orientation times to statistical words and statistical partwords were calculated for each of the 30 participants (see Figure 3). Eleven out of the 15 infants in the

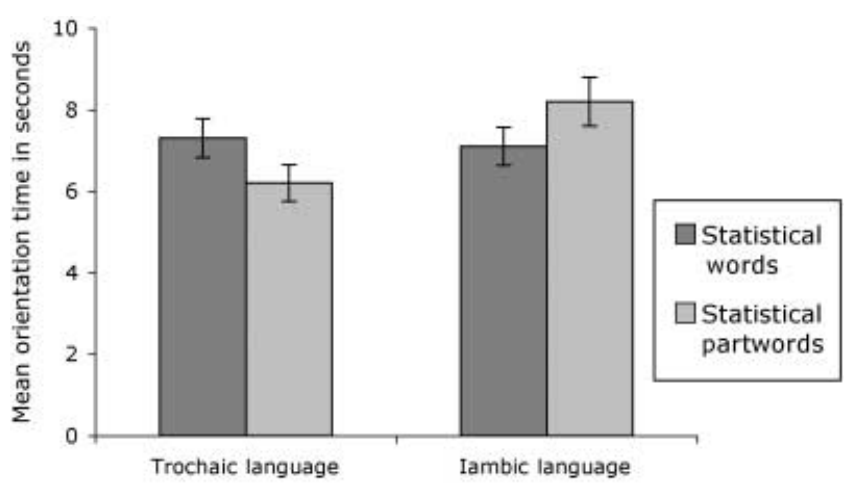

Figure 3 Mean orientation in seconds to statistical words and statistical partwords for Experiment 3. Error bars indicate Standard Error. 
Trochaic Condition listened longer to the statistical words $(M=7.26 ; S D=1.8)$ than to the statistical partwords $(M=6.2 ; S D=1.7)$. This difference was marginally significant by a one-tailed sign test, $p=.06$. Thirteen out of the 15 infants in the Iambic Condition listened longer to the statistical partwords $(M=8.3 ; S D=2.2)$ than to the statistical words $(M=7.1 ; S D=1.8)$. This difference was significant by a one-tailed sign test, $p=$ 004. A 2 (Language Group: Trochaic vs. Iambic) $\times 2$ (Test Item Type: statistical word vs. statistical partword) mixed design ANOVA revealed no main effect of Test Item Type, $F(1,28)=.026, p=.87$, or main effect of Group, $F(1,28)=2.1, p=.16$. However, there was a significant interaction between Group and Test Item Type, $F(1,28)=14.3, p=.0008$. This interaction was driven by longer looking times to statistical words in the Trochaic Condition, $t(14)=2.5, p=.025$, and longer looking times to statistical partwords (or stress words) in the Iambic Condition, $t(14)=2.8, p=.014$. Taken together, these results demonstrate that 11-month-olds segmented out the words defined by the stress cues in the language rather than the syllable transition cues.

\section{General discussion}

Previous studies have demonstrated that English-learners develop the ability to segment iambic words from speech by 11 months of age (Johnson, 2005; Jusczyk et al., 1999b). Although it is generally agreed that 11-montholds' ability to segment iambic words from speech must be linked to their increasing awareness of additional cues to word boundaries, there is still a great deal to be learned about the precise mechanism(s) underlying infants' mastery of the segmentation of iambic words in English. One hypothesis is that as infants increase their repertoire of segmentation cues, developing increasingly accurate segmentation heuristics and becoming word learners, it becomes apparent that stress cues to word boundaries can be inaccurate, i.e. non-initially stressed words exist in English. The ability to segment iambs could lead infants to demote their reliance on stress cues to word boundaries while increasing their reliance on other speech cues to word boundaries, including statistical cues. In this study, we used an artificial language-learning paradigm to examine 11-month-olds' reliance on syllable transition versus stress cues to word boundaries.

In Experiment 1, using the same methodology as Johnson and Jusczyk (2001), we found evidence that 11month-olds still weight stress cues to word boundaries more heavily than statistical cues. In Experiment 3, using the same methodology as Thiessen and Saffran (2003), we replicated these results, i.e. infants segmented trochaic words from speech regardless of whether or not those trochaic words aligned with statistical cues to word boundaries. Taken together, these results suggest that the emergence of the ability to segment iambic words from speech is not linked to a shift in infants' reliance on statistical versus stress cues to word boundaries. But this statement warrants careful consideration. Our finding that 11-month-olds still rely more heavily on stress cues than syllable transition cues does not suggest that 11month-olds have a reliance on stress cues that equals that shown in younger infants. On the contrary, this finding is fully consistent with the hypothesis that 11-month-olds weight stress cues to word boundaries less heavily than 8- and 9-month-olds, but still this decreased reliance on stress cues is not accompanied by an increased reliance on syllable transition cues; in other words, although reliance on stress cues has decreased, the relative ranking of stress and statistical cues remains unaltered, with infants attending more to stress than statistics when segmenting words from running speech. What this means is that an increased reliance on syllable transition cues to word boundaries is unlikely to be the primary mechanism driving 11-month-olds' ability to segment iambic words from speech. Rather, we suspect that the development of this ability is driven by an increased awareness of the individual cues as well as an ability to integrate multiple speech cues in the task of finding word boundaries.

Aside from the discovery of a consistency in the development of the ranking of stress versus syllable distribution cues, the findings reported in this paper highlight another important and perhaps broader reaching issue in the study of early word segmentation. In particular, it is still an open question how artificial language studies translate to natural language settings, given that different segmentation cues carry different weight in different segmentation contexts. For example, artificial language studies typically strengthen the weight of transitional probability cues by presenting words 30 to 60 times within a 2- or 3-minute exposure period. It is likely safe to say that this type of exposure never occurs in the real world, i.e. no natural corpus contains 30 to 60 tokens of each of four words within a single dialogue. At the same time, speech cues such as stress and coarticulation can be more or less salient in artificial languages than in natural languages, depending on the design of the language. The bottom line is that artificial languages allow us to test how infants weight information in the speech stream, but the validity of artificial language studies to test cue weighting hypotheses is dependent upon how accurately the artificial language reflects the structure of the speech signal in the real world, and how likely infants' behavior in the experiment is to reflect real language processing skills as opposed to laboratory-induced strategies.

The weighting of stress versus syllable transition cues has not, to our knowledge, been directly tested with natural speech stimuli (see Christiansen, 1998, for work modeling cue integration), but there is evidence to suggest that syllable transition cues are not strongly weighted by 11month-olds. In Jusczyk et al. (1999b), 11-month-olds were familiarized with passages of cross-word sequences of syllables that repeatedly co-occurred (e.g. guitar was always followed by $i s$ ). In this case, infants did not extract the co-occuring cross-word trochaic sequence taris as a 
word. Rather, they extracted the iambic word guitar from the speech stream. This does not demonstrate that 11-month-olds weight stress cues more heavily than statistical cues. Rather, it suggests that the infants in this study used a combination of (other) cues (e.g. coarticulation and allophonic cues; the fact that 'is' is a common function word; the prosodic markers of the phrase boundary between the subject 'guitar' and the verb 'is') to arrive at the correct segmentation of the passage containing a non-initially stressed target word. Importantly, this study also shows that 11-month-olds weight a constellation of other segmentation cues more heavily than either stress or statistical cues taken on their own. Indeed, there is evidence that prosodic boundaries (Gout, Christophe \& Morgan, 2004; Seidl \& Johnson, 2006, 2008) and function words (Shi, Werker \& Cutler, 2006) play an important role in early word segmentation behaviors. Additional studies with natural language stimuli that pit one segmentation cue against another are needed to fully understand infants' transition from immature to more mature word segmentation strategies. Johnson (2008) took an important step towards addressing these issues by replicating Jusczyk et al.'s finding with 12-month-olds and more tightly controlled stimuli. Johnson's stimuli differed from Jusczyk et al.'s stimuli in that the syllables constituting the cross-word sequences were never separated by phrase boundaries, and the cross-word syllable sequence did not contain embedded function or auxiliary words (e.g. the cross-word sequence dogma occurred in the phrase dog maligns). This study demonstrated that infants familiarized with passages containing the phrase dog maligns did not extract the word dogma, whereas infants familiarized with passages containing the phrase dogma lines did. Johnson interpreted these findings as evidence that fine-grained acoustic phonetic cues to word boundaries are likely to be a powerful driving force behind infants' development of the ability to segment iambic words from speech. This interpretation fits well with our suggestion that sensitivity to segmentation cues other than syllable transition enables infants to first begin segmenting words from speech. This view is also supported by evidence that adult listeners find acoustic-phonetic cues to be a powerful indicator of word boundaries (Salverda, Dahan \& McQueen, 2003; Shatzman \& McQueen, 2006a, 2006b).

The natural language studies described above suggest that older infants are not highly reliant on syllable transition cues to word boundaries. Although there are no natural language studies directly testing stress versus syllable transition cues, there are natural language studies looking at stress versus other segmentation cues. For example, there is evidence to suggest that 9-month-olds weight stress cues more heavily than phonotactic cues (Mattys et al., 1999). It would be useful to know if 11-month-olds behave similarly. Another interesting approach to understanding the development of segmentation skills would be to test 11-month-olds on an artificial language containing conflicting allophonic and stress cues to word boundaries. Or perhaps to test infants on a language containing phonotactic and allophonic cues indicating one segmentation and stress cues indicating another. Finally, if we are to ever develop a truly language-general theory of developmental word segmentation, researchers must eventually consider cue re-ranking in Englishlearning versus non-English-learning children, and in monolingual versus multilingual children.

In conclusion, our results do not support the notion that greater reliance on syllable transition cues enable infants to overcome their reliance on stress cues to find word boundaries in fluent speech. At the same time, our findings do not rule out the possibility that 11-montholds rely less heavily on stress cues than other speech cues to segment words from speech. In fact, we believe that this is likely to be the case in that greater reliance on (or integration of) other speech cues must play a causal role in infants' realization that non-initially stressed syllables can be word onsets. But it is still unclear how infants go about integrating cues in the task of word segmentation and whether acoustic cue weighting, which changes over time, may influence the integration of these cues. For example, developmental work by Nittrouer and colleagues (1992; Nittrouer \& Miller, 1997) suggests developmental weighting shifts between childhood and adulthood. Further, Seidl (2007; Seidl \& Cristià, 2008) suggests that infants may have biases in attention to different acoustic cues but that shifts in weighting may occur at an age at which infants start to be able to use cues separately. Only further research using a wide variety of stimuli and testing methodologies will enable us to better understand how word segmentation strategies develop in young infants.

\section{Acknowledgements}

We would like to thank Amanda Klein, Ashley Casey, Alejandrina Cristià and Ilana Feld for help in running and recruiting subjects. In addition, we thank Kristine Onishi for useful comments on an earlier draft of this paper. Funding for this research was provided by a Research Grant from NICHD (R03 HD046463-0) as well as funds from Purdue University to AS. Additional funding was provided by the Max Planck Institute for Psycholinguistics and a Spinoza Grant awarded to Anne Cutler by NWO.

\section{References}

Aslin, R.N. (2007). What's in a look? Developmental Science, 10, 48-53.

Aslin, R.N., Saffran, J.R., \& Newport, E.L. (1998). Computation of conditional probability statistics by 8 -month-old infants. Psychological Science, 9, 321-324.

Bortfeld, H., Morgan, J., Golinkoff, R., \& Rathbun, K. (2005). Mommy and me: familiar names help launch babies into speech-stream segmentation. Psychological Science, 16 (4), 298-304. 
Brent, M.R., \& Siskind, J.M. (2001). The role of exposure to isolated words in early vocabulary development. Cognition, 81, 33-44.

Christiansen, M.H., Allen, J., \& Seidenberg, M.S. (1998). Learning to segment speech using multiple cues: a connectionist model. Language and Cognitive Processes, 13, 221-268.

Cole, R.A., \& Jakimik, J. (1980). A model for speech perception. In R. Cole (Ed.), Perception and production of fluent speech (pp. 136-163). Hillsdale, NJ: Erlbaum.

Curtin, S., Mintz, T.H., \& Christiansen, M.H. (2005). Stress changes the representational landscape: evidence from word segmentation. Cognition, 96, 233-262.

Cutler, A., \& Butterfield, S. (1992). Rhythmic cues to speech segmentation: evidence from juncture misperception. Journal of Memory and Language, 31, 218-236.

Cutler, A., \& Carter, D.M. (1987). The predominance of stressed initial syllables in the English vocabulary. Computer Speech and Language, 2, 133-142.

Gerken, L., \& Zamuner, T. (2004). Exploring the basis for generalization in language acquisition. Paper presented at LabPhon 9, Illinois.

Gout, A., Christophe, A., \& Morgan, J. (2004). Phonological phrase boundaries constrain lexical access: II. Infant data. Journal of Memory and Language, 51, 547-567.

Hayes, J.R., \& Clark, H.H. (1970). Experiments in the segmentation of an artificial speech analogue. In J.R. Hayes (Ed.), Cognition and the development of language (pp. 221-223). New York: Wiley.

Hunter, M.A., \& Ames, E.W. (1988). A multifactor model of infant preferences for novel and familiar stimuli. In C. RoveeCollier \& L.P. Lipsitt (Eds.), Advances in infancy research (Vol. 5, pp. 69-95). Norwood, NJ: Ablex.

Johnson, E.K. (2005). English-learning infants' representations of word forms with iambic stress. Infancy, 7, 95-105.

Johnson, E.K. (2008). Infants use prosodically conditioned acoustic-phonetic cues to extract words from speech. Journal of the Acoustical Society of America, 123 (6), E144-E148.

Johnson, E.K., \& Jusczyk, P.W. (2001). Word segmentation by 8-month-olds: when speech cues count more than statistics. Journal of Memory and Language, 44, 548-567.

Jusczyk, P.W. (1997). The discovery of spoken language. Cambridge, MA: MIT Press.

Jusczyk, P.W., \& Aslin, R.N. (1995). Infants' detection of sound patterns of words in fluent speech. Cognitive Psycho$\log y, 29,1-23$.

Jusczyk, P.W., Hohne, E.A., \& Bauman, A. (1999a). Infants' sensitivity to allophonic cues for word segmentation. Perception and Psychophysics, 61, 1465-1476.

Jusczyk, P.W., Houston, D., \& Newsome, M. (1999b). The beginnings of word segmentation in English-learning infants. Cognitive Psychology, 39, 159-207.

Jusczyk, P.W., Luce, P., \& Charles-Luce, J. (1994). Infants' sensitivity to phonotactic patterns in the native language. Journal of Memory and Language, 33, 630-645.

Kuhl, P.K. (2000). A new view of language acquisition. Proceedings of the National Academy of Sciences, 97 (22), 11850-11857.

McQueen, J.M. (1998). Segmentation of continuous speech using phonotactics. Journal of Memory and Language, 39, 21-46.
Mattys, S.L., \& Jusczyk, P.W. (2001). Phonotactic cues for segmentation of fluent speech by infants. Cognition, 78, 91121.

Mattys, S.L., Jusczyk, P.W., Luce, P.A., \& Morgan, J.L. (1999). Phonotactic and prosodic effects on word segmentation in infants. Cognitive Psychology, 38, 465-494.

Nittrouer, S. (1992). Age-related differences in perceptual effects of formant transitions within syllable and across syllable boundaries. Journal of Phonetics, 20, 351-382.

Nittrouer, S., \& Miller, M. (1997). Predicting developmental shifts in perceptual weighting schemes Journal of the Acoustical Society of America, 101, 2253-2266.

Saffran, J.R., Aslin, R.N., \& Newport, E.L. (1996). Statistical learning by 8-month-old infants. Science, 274, 1926-1928.

Salverda, A.P., Dahan, D., \& McQueen, J.M. (2003). The role of prosodic boundaries in the resolution of lexical embedding in speech comprehension. Cognition, 90, 51-89.

Seidl, A. (2007). Infants' use and weighting of prosodic cues in clause segmentation. Journal of Memory and Language, 57, $24-48$.

Seidl, A., \& Cristià, A. (2008). Developmental changes in the weighting of prosodic cues. Developmental Science, 11, 596606.

Seidl, A., \& Johnson, E.K. (2006). Infant word segmentation revisited: edge alignment facilitates target extraction. Developmental Science, 9, 565-573.

Seidl, A., \& Johnson, E.K. (2008). Perceptual factors influence infants' extraction of onsetless words from continuous speech. Journal of Child Language, 35, 1-24.

Shatzman, K.B., \& McQueen, J. (2006a). Prosodic knowledge affects the recognition of newly-acquired words. Psychological Science, 17 (5), 372-377.

Shatzman, K., \& McQueen, J. (2006b). The modulation of lexical competition by segment duration. Perception and Psychophysics, 68, 1-16.

Shi, R., Werker, J., \& Cutler, A. (2006). Recognition and representation of function words in English-learning infants. Infancy, 10 (2), 187-198.

Swingley, D. (2005). Statistical clustering and the contents of the infant vocabulary. Cognitive Psychology, 50, 86-132.

Thiessen, E.D., Hill, E.A., \& Saffran, J.R. (2005). Infant-directed speech facilitates word segmentation. Infancy, 7, 53-71.

Thiessen, E.D., \& Saffran, J.R. (2003). When cues collide: use of stress and statistical cues to word boundaries by 7- to 9month-old infants. Developmental Psychology, 39, 706-716.

Thiessen, E.D., \& Saffran, J.R. (2004). Spectral tilt as a cue to word segmentation in infancy and adulthood. Perception and Psychophysics, 66, 779-791.

Thiessen, E.D., \& Saffran, J.R. (2007). Learning to learn: infants' acquisition of stress-based segmentation strategies. Language Learning and Development, 3, 73-100.

Tyler, M.D., \& Johnson, E.K. (2006). Testing the limits of artificial language learning. Poster presented at the Fifteenth International Conference on Infant Studies, Kyoto, Japan.

Werker, J.F., \& Tees, R.C. (1984). Evidence for perceptual reorganization during the first year of life. Infant Behavior and Development, 7, 49-63.

Received: 30 June 2006

Accepted: 6 January 2008 